\section{TREATMENT OF TROCHANTERIC FRACTURES OF FEMUR}

\author{
BY
}

\author{
P. A. RING, M.S., F.R.C.S. \\ Consultant Orthopaedic Surgeon, Redhill (Surrey) \\ Group of Hospitals
}

Fractures of the femur in the region of the trochanters are common injuries in the elderly and occasionally occur in young adults and children. In some cases the damage is confined to an avulsion of the lesser or greater trochanter or to a crack which passes through part of the trochanteric region but spares the calcar femorali, and for these patients little but rest in bed for a few days is required. When the continuity of the femur is lost the injury is a severe one and walking is impossible. Deformity and pain are often considerable, and, in contrast with the high fracture of the femoral neck, delay in diagnosis is uncommon.

In any assessment of the value of treatment in this type of injury consideration must be given to the cases which are received. Residents of nursing-homes and geriatric wards and those living in mental institutions or being cared for in bed by their relatives at home have only a tenuous hold on life, and if they fall the difficulties of restoring them to their preoperative level of existence are very great. These are the patients who tend to fill the wards of the large provincial hospitals and whose stay is out of proportion to the severity of their injury. Those who are living at home, active within the limits of their age, rarely present major problems of medical care and can usually be restored to their life in the community provided that their stay in hospital is not prolonged.

In most centres a trochanteric fracture is not regarded as an emergency, and a day or two is often considered well spent in attempting to improve the general condition. During this time the leg may be immobilized between sand-bags or put on skin traction. Continuous traction is occasionally held to be the treatment of choice in some trochanteric fractures, and patients treated in this way may stay in bed for up to four months. It is my view that the pain of the injury alone would justify internal fixation of the fracture, and when to this is added the greater ease of nursing and the possibility of early ambulation, the advantages of surgical treatment of this fracture must outweigh any small risk there may be from the anaesthetic and the operation. This should therefore be performed as soon after admission as possible. Even when the condition of the patient is poor there is nothing to be gained by delay. It is rare to find a patient who is unfit for a general anaesthetic and even rarer to find one whose condition will improve before the fracture has been secured.

The reduction of this injury is best done upon an orthopaedic table, but the varying types of lesion may require slightly different procedures. In the pertrochanteric fracture the proximal fragment is internally rotated and flexed and the leg must be internally rotated firmly before the fracture is reduced. In the subtrochanteric fracture the proximal fragment is flexed and abducted by the psoas and abductor muscles and reduction can be achieved only by flexing the limb and externally rotating it upon the traction table. In the intertrochanteric lesion, particularly when the lesser trochanter is avulsed, internal rotation and flexion are normally required.

Fixation throughout this group of cases has been achieved by the Jewett nail. In inserting this appliance a large cortical hole must be made so that the nail can be introduced without fragmentation of the fracture site, and its length must be such that its tip extends to within $\frac{1}{2}$ in. $(1.3 \mathrm{~cm}$.) or less of the joint margin. Should the nail enter the joint little discomfort is experienced, and only in the younger patient will it subsequently require removal. With a range of nails varying by $\frac{1}{2}$ in. $(1.3 \mathrm{~cm}$.) between the lengths of 3 and 5 in. ( 7.5 and $12.5 \mathrm{~cm}$.) and with 10-degree angle variations between 120 and 150 degrees, a total stock of 20 nails must be held in order to secure almost any fracture. In a subtrochanteric lesion it is of advantage to have a longer plate on the nail, and for this purpose a modified appliance is available.

The purpose of operation is to secure the fracture so firmly that the patient may be mobilized immediately and permitted to bear weight upon the injured leg. It is rare to find a fracture which cannot be secured in this way and in which early weight-bearing is impossible, but in the obese patient with a severely comminuted lesion a short period of skeletal traction may be desirable. Early weight-bearing is painful for a few days, but most patients will walk independently with a frame within a week and will be confident on two sticks a week or two later. It has been shown by Sevitt and Gallagher (1959) that femoral thrombosis and pulmonary embolism are common in this group of patients. We have therefore administered phenindione, except to those patients with a history of peptic ulceration or other lesions likely to bleed, with liver failure, or with urinary infection.

\section{Present Series}

The patients in the present series were admitted during the years 1960 and 1961. They include all patients admitted to three general hospitals and comprise substantially the entire incidence of the injury in this area. From the patients who have been treated only those from the surrounding mental hospitals have been excluded, because the follow-up of these patients has not been adequate and their mental condition has often made formal treatment impossible. During the years in question 149 patients with fractures of the femoral neck were admitted and 69 of these sustained trochanteric fractures. These patients tend to be slightly older than those with a mid-cervical or subcapital lesion, although the difference between the two groups is not great. The details of each operation and the condition of the patients on review were recorded upon a punch card and each patient was kept under supervision until the fracture was fully united. Nonunion and avascular necrosis are very rare after fractures in this region and their fate can usually be determined at the end of six months.

There were 18 deaths in the 69 patients treated in this way, an overall mortality of $26 \%$. During the same period 12 of the 80 patients with midcervical and subcapital fractures died, a mortality of $15 \%$. These differences reflect the slightly greater age of the patient with a trochanteric fracture and the greater severity of the injury. The causes of death in these patients are recorded in Table I. It must be accepted that in some 
cases this cause of death is no more than a terminal event in which senility, pain, respiratory infection, and occasionally pressure sores play a part, and those patients whose cause of death is certified as bronchopneumonia merely fail to show evidence of any other specific disease.

TABle I.-Cause of Death in 18 Patients

\begin{tabular}{|c|c|c|}
\hline $\begin{array}{c}\text { No } \\
\text { Anticoagulants }\end{array}$ & $\begin{array}{c}\text { Under Anticoagulant } \\
\text { Treatment }\end{array}$ & $\begin{array}{l}\text { After Anticoagulant } \\
\text { Withdrawal }\end{array}$ \\
\hline $\begin{array}{l}\text { Pulmonary embolism } 2 \\
\text { Bronchopneumonia } 9\end{array}$ & 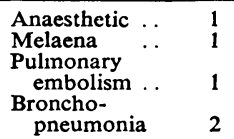 & Coronary thrombosis 2 \\
\hline
\end{tabular}

There were two deaths from pulmonary embolism in patients not on anticoagulant treatment and one in a patient whose anticoagulant treatment appeared to have been adequate and was still being maintained at the time of death. One patient's death was directly attributable to haemorrhage from the bowel, and in two others the withdrawal of anticoagulants, although gradual, was followed by a fatal coronary thrombosis. No purpose can be served by comparing the mortality of patients on anticoagulant treatment with that of patients who were not, since the latter probably included a greater number of patients whose general condition was poor owing to associated disease.

The average stay in hospital of the 51 survivors of the trochanteric group was 26 days and more than half of these patients went home, walking, bearing weight and using one or two sticks, within three weeks of the day of their injury. Many of the others stayed longer only because their home conditions were unsuitable and a few because they were awaiting a place in a geriatric rehabilitation unit: At the end of six months all but one of the fractures were firmly united by bone, the solitary non-union being in a patient walking on a nailplate without difficulty or discomfort. No other example of delay in union was seen, and during the limited follow-up performed there was no case of avascular necrosis. One pin inserted in a heavy patient with a leg paralysed from poliomyelitis bent, but the fracture united without significant deformity. There was no other example of metal failure or plate fracture.

In the severely comminuted fracture some displacement of the fragments tended to occur before union became complete, and if a perfect anatomical result is important in a comminuted fracture it may occasionally be necessary to supplement internal fixation by skeletal traction. There has, however, been no displacement of any magnitude within this series and no patient in whom the shortening or abduction was such that a raised shoe became necessary. Penetration of the acetabulum by the pin occasionally occurred in a comminuted fracture and was usually due to shortening of the femoral neck as union progressed. Little discomfort occurred even when the pin projected $\frac{1}{4}$ in. $(6 \mathrm{~mm}$.) or more beyond the femoral head and in most patients this penetration could be ignored. There was no example of pin movement or of displacement within the head, other than along its own track as the fracture united.

\section{Anticoagulants}

The prophylactic use of anticoagulants in fractures of the femoral neck was suggested by Sevitt and
Gallagher (1959), who in a well-controlled trial showed that femoral thrombosis was reduced from $28.7 \%$ in the controls to $2.7 \%$ in the phenindione series; and embolism, present in $18 \%$ of controls and fatal in $10 \%$, did not occur in any patient on phenindione. The overall mortality in the phenindione series was $16.6 \%$ and in the controls $28 \%$. These differences are impressive and treatment with phenindione was therefore used in all patients other than those with wellrecognized contraindications. It proved extremely difficult to maintain an adequate prothrombin level, and the dosage required fluctuated a great deal from patient to patient and from day to day. After two to three weeks the dosage was relatively easy to standardize, but during the first week or two the prothrombin index sometimes fluctuated widely, and, even in spite of a relatively high index at times, bleeding sometimes occurred. Wound haematomas were common and occasionally became large enough to require evacuation. Infection of the haematoma was present in a few cases, but was severe in only one case. Mild or moderate haematuria with associated urinary infection and sometimes with retention occurred in several patients and severe bowel haemorrhage, from which the patient died, in one.

The total numbers on anticoagulant treatment, and the mortality, are recorded in Table II. Two patients who did not receive anticoagulant treatment died of emboli, and no emboli were recorded in the group treated with phenindione. Two patients developed coronary thrombosis after cessation of anticoagulant

TABLE II.-Relationship of Mortality to Anticoagulant Treatment

\begin{tabular}{|c|c|c|}
\hline & Phenindione & No Phenindione \\
\hline $\begin{array}{ll}\text { Total No. of cases } & \ldots \\
\text { Deaths } & . .\end{array}$ & $\begin{array}{r}41 \\
7\end{array}$ & $\begin{array}{l}28 \\
11\end{array}$ \\
\hline
\end{tabular}

treatment although this had been tailed off gradually. The problems of phenindione treatment in these patients seemed to be formidable and it is my impression that the wound haematomas, urinary bleeding, and occasional wound infection were delaying the progress of patients whose fractures were well secured and were often prejudicing the regime of early ambulation. Patients making good progress for a few days sometimes developed a haemorrhage which might in a healthy person be trivial, but which involved in these patients a further period of rest in bed for a few days with the associated deterioration that so often occurs.

One must consider whether the reduction in mortality from embolism which occurs during phenindione treatment is worth an increase in morbidity. One must also ask whether such treatment is in fact necessary when the patient is mobilized within a day or two of the fracture occurring. It is worth bearing in mind that in the series reviewed by Sevitt and Gallagher (1959) half the patients waited two or more days after admission before operative treatment, a feature which must predispose to the very high rate of femoral thrombosis and of pulmonary embolism which they record in their control series. It seems likely that, with early operation and immediate weight-bearing, the likelihood of significant femoral thrombosis may be small and that the dangers of anticoagulant treatment may be greater than those of venous thrombosis. 
Eskeland (1962) has pointed out that fatal pulmonary embolism is rare in patients below the age of 75 with fractures of the femoral neck and has suggested that anticoagulant treatment might be resserved for this group. This is, however, the group in which control is so difficult and in which the complications of anticoagulant treatment are most common. While there can be little doubt that the mortality from embolism can be reduced in this way, it seems likely that early operation and early weight-bearing may achieve a similar effect without the risks which anticoagulant treatment involves. In these patients such a regime may make prophylactic anticoagulants unnecessary.

\section{Conclusions}

The merits of early operation in a patient with a trochanteric fracture are almost entirely self-evident, but it is still surprising how many of these patients wait for some days before the fracture is secured. Most of the injuries in this series had been treated within 24 hours. Delay has usually been either in diagnosis or in arranging for admission. Occasionally an elderly woman living alone may fall and remain unable to move until she is discovered by her neighbours a day or two later.

There appears to be nothing to be gained from delaying the operation to correct medical defects. Few of these patients might be regarded as fit for their age, but most of their disabilities will not improve by a few days' medical treatment, and it is almost always preferable to secure the fracture first and afterwards to engage in such medical treatment as is appropriate.

It is doubtful whether there is any trochanteric fracture which cannot be secured by internal fixation, and splintage of any other sort is normally unnecessary. The relief of pain and the associated improvement in general condition are so marked that even in a patient who was not ambulant before the fracture occurred operation is always worth performing.

Internal fixation should normally be secure enough to permit immediate weight-bearing, and it is rare indeed to find a patient in whom this cannot be achieved. After a day or two of discomfort these patients progress rapidly to walking with a frame and later with sticks, and half the survivors can be expected to return to their own homes within three weeks of the accident.

The dangers of anticoagulant treatment in the elderly are formidable, and it is doubtful whether its value in preventing thrombosis and embolism outweighs its dangers.

\section{Summary}

Sixty-nine trochanteric fractures of the femur have been treated by immediate fixation with a Jewett nailplate, early weight-bearing, and anticoagulants.

There were 18 deaths in this series, giving a mortality of $26 \%$. The average stay in hospital in the survivors was 26 days, and more than half of them went home bearing weight, using one or two sticks, within three weeks of the injury.

Most of these patients received prophylactic phenindione treatment during the post-operative period. It seems likely that the risks of anticoagulant treatment in these patients are greater than the risks of femoral thrombosis and embolism.
There was no complication attributable to immediate weight-bearing, and the anatomical reconstitution of the femoral neck and upper femoral shaft was uniformly good.

REFERENCES

Eskeland, G. (1962). Lancet, 1, 1035

Sevitt, S., and Gallagher, N. G. (1959). Ibid., 2, 981.

\section{ANTINUCLEAR FACTOR AND OTHER ANTIBODIES IN BLOOD AND LIVER DISEASES}

BY

\section{E. J. HOLBOROW, M.D.}

G. L. ASHERSON,* B.M., B.Ch., M.R.C.P.

G. D. JOHNSON, F.I.M.L.T.

Rheumatism Research Unit (M.R.C.), Canadian Red Cross Memorial Hospital, Taplow, Maidenhead, Berkshire

AND

R. D. S. BARNES, M.B.

Department of Clinical Pathology

D. S. CARMICHAEL, M.B.

Department of Haematology

Guy's Hospital, London

Auto-antibodies have been demonstrated in systemic lupus erythematosus, Sjögren's disease, rheumatoid arthritis, and other conditions. They include the antinuclear factors, complement-fixing antibodies against liver and other organs, the rheumatoid factor, and antibodies against red cells (see Hijmans, Doniach, Roitt, and Holborow, 1961). As immunological mechanisms are thought to play a part in certain blood and liver diseases it was of interest to determine the incidence of antibodies in these conditions.

\section{Materials and Methods}

Selection of Cases.-Patients with blood diseases, including pernicious anaemia, haemolytic anaemia, thrombocytopenia, agranulocytosis, leukaemia, Hodgkin's disease, lymphosarcoma, multiple myeloma, and infectious mononucleosis, seen by the pathologist during six months were examined as well as sera from normal subjects. The miscellaneous group in the accompanying Table included single cases of anaemia due to folic-acid deficiency without steatorrhoea, postgastrectomy anaemia, anaemia with steatorrhoea, familial acholuric jaundice, auto-immune haemolytic anaemia, anaemia secondary to uraemia, idiopathic aplastic anaemia, agranulocytosis possibly due to sulphonamides, anaemia secondary to carcinoma of the stomach, anaemia secondary to subacute bacterial endocarditis, and haemophilia. The patients with infectious mononucleosis had Downey cells and a positive Paul-Bunnell reaction. Patients with liver disease were selected on the basis of abnormal liver-function tests and clinical evidence of hepatobiliary disorder. The patient with scleroderma was included because she presented with agranulocytosis. The following laboratory tests were read without knowledge of the diagnosis.

Fluorescent Test for Antinuclear Factor.-The indirect technique was used (Weir, Holborow, and Johnson, 1961).

*Beit Memorial Fellow. 